\title{
An Analysis of English and Chinese Numerical Idioms Sharing the Equivalent Connotations*
}

\author{
Yuan Kong \\ Department of Foreign Languages, Jining Medical University, Jining, China
}

\begin{abstract}
This paper, based on the analysis of English and Chinese numerical idioms which share the equivalent or similar connotative meaning, according to the different corresponding relationship between the expressive means and expressive forms, are divided into four groups. The expressive means are classified in terms of rhetorical device of English and Chinese numerical idioms and considered mainly from vehicle. Because the rhetorical device mainly used in number and numerical idiom is metaphor, and as the cultural backgrounds are different, the metaphorical image varies a lot. Sometimes, the same image is endowed different emotional colors in different countries and used in different occasions. Metaphorical images in English and Chinese numerical idioms are also reflections of the historical traditions, lifestyles, values, moral standards and customs in languages.
\end{abstract}

Index Terms - English, Chinese, numetical idiom, connotation

\section{INTRODUCTION}

Connotative meaning is the reflection of essential attribute and image of the phenomenon of the outside world in semantics. It reflects the people's subjective attitudes and emotions, which is closely related to the national cultural background (Zhao \& Jiang, 2003). When expressing the same semantics, there exist similarities or identities in different languages, that is, the forms of expression are the same or similar. Idioms, sharing the equivalent or similar expressions and connotations, can be perceived from the literal meaning directly. There are still some other numerical idioms in English and Chinese having the equivalent or similar connotative meaning but differing in expressive means and expressive forms. So we can find that the essential differences between English and Chinese numerical idioms are not the content expressed by them, but the expressive forms and expressive means.

\section{Rhetorical DeVICES OF THE Numbers In ENGLISH AND CHINESE IDIOMS}

Numbers are usually used to indicate exact number and amount, its notable feature is the accuracy of expression of concept. In the practical language use, numbers are always used to express abstract rather than concrete concept. In English some numbers have two kinds of applications: on the one hand, they are pure accurate numbers and have the feature of accuracy; on the other hand, they may be used as fuzzy ones to express the amount that is not so exact and have the feature of fuzziness. For example, "hundred, thousand, million" means "large amount of", but how much or how many, it is not clear. That is what we call fuzzy numbers. This kind of semantic ambiguity endows certain numbers with rhetorical functions, thus they are often used in the rhetorical devices such as hyperbole, metaphor, metonymy, euphemism and so on. Numbers are the important aids to characters. Usually, they are inseparable from various types of articles in describing quantity, degree, frequency, scale, level of things and other aspects. Exact numbers can express something more accurately and powerfully. Therefore, numerical idioms have the rhetorical features such as analogy, exaggeration and euphemism. If they are used appropriately, the language will become more powerful and efficient.

First, let us have a brief look at some rhetorical devices of the numbers in English and Chinese idioms.

\section{Metaphor}

Metaphor is a figure of speech in which an expression is used to refer to something that it does not literally denote in order to suggest a similarity. Unlike simile, metaphors state that something is something else, which has no "like" or "as" in the comparison. Metaphor is or was also occasionally used to denote rhetorical figures of speech that achieve their effects via association, comparison or resemblance (e.g. antithesis, hyperbole, metonymy and simile, which are then all considered types of metaphor). Aristotle used both this sense and the regular, current sense above. It is often used to make the writing more interesting or entertaining.

In some cases, the numbers used in numerical idioms often lose their specific meaning and evolve into the meaning associated with something or possessing the similar characteristics of something. For example: "a hundred to one" (very likely or pretty sure); "a fall between too stool" (fail to obtain anything at last); "be like two peas in a pod" (much like).

Hyperbole

Hyperbole is a figure of speech that uses an exaggerated or extravagant statement to evoke strong feelings or create a

\footnotetext{
* Funding Source: JMC Scientific Research Project (JY2013RW032)
} 
strong emotional response. As a figure of speech it is not intended to be taken literally and is frequently used for humor. Hyperbole is an exaggeration to create emphasis or effect. As a literary device, hyperbole is often used in poetry, and is frequently encountered in casual speech. An example of hyperbole is "The bag weighed a ton". Here it helps to make the point that the bag was very heavy, although it is not probable that it would actually weigh a ton.

Exaggerated numbers could "win more with fewer" and have a more vivid, intuitive effect. English numbers such as "hundred, thousand, million, nine, ten and twenty" are used for exaggeration. For example: "one in a thousand" (choosing one from one hundred); "a nine day's wonder" (good things or sights disappear soon after their brilliant appearance).

\section{Metonymy}

Metonymy is a figure of speech used in rhetoric in which a thing or concept is not called by its own name, but by the name of something intimately associated with that thing or concept. For instance, "Westminster" is used as a metonym (an instance of metonymy) for the Parliament of the United Kingdom, because it is located there.

Metonymy works by the contiguity (association) between two concepts. When it is used, it does not typically wish to transfer qualities from one referent to another as they do with metaphor. Thus, metonymy works by calling up a domain of usage and an array of associations. Metonymy can also refer to the rhetorical strategy of describing something indirectly by referring to things contiguous to it, in either time or space.

In digitization times, more and more numbers become popular in slang expressions. For example, five by five (very short and overweight figure); five-and-ten (the store in which cheap goods are sold). In Chinese metonymy, the target persons or things are replaced by something associated closely with them. For example, in the Chinese idiom "si4" mian4 bal fang1" (all sides), the Chinese character "si4" (four) and "bal" (eight) refers to all directions.

Euphemism

Euphemism is a substitution for an expression that may offend or suggest something unpleasant to the receiver, using instead an agreeable or less offensive expression, or to make it less troublesome for the speaker. Some euphemisms are intended to amuse, while others are created to mislead. Here are some examples: "four eyes" (shortsighted); "five fingers" (stealer); "three hands" (stealer) and so on.

\section{Repetition}

Repetition is the simple repeating of a word within a sentence or a poetical line in order to emphasize. This is such a common literary device that it is almost never even noted as a figure of speech. There are also some examples in both English and Chinese idioms. For example: "twenty and twenty" (a huge number of something that can not be counted); "qian1 qian1 wan4 wan4" (tens of thousands); "san1 san1 liang3 liang3" (twos and threes).

\section{Contrast}

An author writes contrast when he or she describes the difference(s) between two or more entities or between two sides of the same thing. As a kind of rhetorical devices, "contrast" in English is the same as "dui4 zhao4" (contrast) in Chinese. Take some English numerical idioms for example: "Two sparrows on one ear of corn make an ill agreement"; "Three Stooges is a great space"; ect.

\section{Classification of Numerical Idioms BASED On the EXPRESSIVE Structures AND MEANS}

The greatest differences in terms of figures of speech of English and Chinese due to cultural differences - different people of the same world have different angles of view or perspectives, which will produce different vehicles that are used to image similar phenomenon. Therefore, the following part will be discussed mainly on the basis of the differences of vehicles in Metaphors.

\section{A. Numerical Idioms with Equivalent Expressive Structures and Means}

Numerical idioms, sharing the same or similar expressions and connotations, can be perceived from the literal meaning directly.

1. Adoption of the same number

The contents expressed by both English and Chinese numerical idioms are equivalent, the expressive means and structures are quite corresponding, and the numbers chosen are the same. We name such correspondence as semantical correspondence of both English and Chinese numerical idioms. In English, the idioms sharing the equivalent structure and meaning rarely exist, but there are still some. For example:

(1) Four-eyes

si4 (four) yan3 (eyes) er

Because people have the same physiological characteristics, one will wear eye-glasses if he is myopia. The person wearing eye-glasses just like having four eyes, so both idioms in English and Chinese are used to express the person who is wearing eye-glasses, and they are considered as an impolite expression.

(2) Second hand

er4 (second) shou3 (hand)

\footnotetext{
${ }^{1}$ In this article, for the purpose of an easier and labor-saving intercultural communication, all the Chinese characters are represented by their pronunciations, that is, Chinese "pinyin". The numbers from 1 to 4 stand for the four kinds of Chinese tones.
} 
The idiom "second hand" is used to express the meaning that something has already been used and worn. The corresponding Chinese form is "er4 shou3". Compared with the new-bought goods, "second-hand" refers to the goods that have been bought once or twice. Now it can be used to refer to things that are not got directly. Sometimes it refers to the news, or service, meaning not being the latest. Recently there are more and more words using "second-hand", then some new meanings come into being. For example, "second-hand smoking"/ er4 shou $3^{2}$ (second-hand) yan 1 (smoking) (smell the smoke from the smoking person), "second-hand fragrance"/ er4 shou3 (second-hand) xiang1 (fragrance) (meaning the fragrance is too strong that the people around feel uncomfortable). These are summaries of people's common life experience.

There are some other examples of numerical idioms that have the equivalent or similar meanings, such as "nine to five/ zhao1 jiu3 (nine) wan3 wu3 (five)", "one ray of hope/ yil (one) xian4 xi1 wang4", "He that will thrive must rise at five/ wu3 (five) geng1 qi3 chuang2, bai3 shi4 xing1 wang4", "in at one ear and out at another/ yi1 zhi1 (one) er3 duo1 jin4, yi1 zhi1 (another) er3 duo1 chu1" and so on.

As we all know, human being has many same or similar experiences no matter what kind of nationality or skin he has, and no matter where he lives. They have the similar life necessities, lifestyles, thoughts and feelings. These similarities determine that the expressive contents, structures and means are very similar, or even the same.

2. Adoption of the different number

The contents expressed by both English and Chinese numerical idioms are equivalent, the expressive means and structures are quite corresponding, but the difference lies in the choice of the numbers.

(3) Six of one and half a dozen of the other

ban4 (half) jin1 ba1 (eight) liang3 (half a catty and eight taels)

This means six is the same to half a dozen. This is originated from duodecimal system. Duodecimal system was brought by Dane when it conquered England and is used still nowadays. From the numerical idiom we can find Dane's custom: because one dozen is twelve, a half dozen is six. So the idiom both in English and Chinese is very similar. In ancient China, one catty is sixteen taels, one tael is a star, and sixteen taels are sixteen stars. They are consisted of Triones, Southern Dipper, and the three gods of fortune, prosperity and longevity. If the sellers give the customers enough, the scale will reach the three gods of fortune, prosperity and longevity (Ma, 2004). So 8 taels is half a catty. Afterwards this word evolved into the meaning that the weight is the same among strength, ability and authority. We should pay attention that Chinese "ban4 jin1 ba1 liang3" is always used to express derogatory meaning.

(4) Five-and-dime store

liang3 (two) yuan2 dian4 (the store where the goods are sold only at the price of two yuan)

In English "five-and-dime store" is the store in which cheap goods are sold. In "five-and-dime", "five" refers to "five cents", dime refers to "ten cents". "Five-and-dime store" in America just like some stores in which goods are sold at 8 yuan or 10 yuan in Hong Kong. In such a store, the goods are sold at 5 cents or 10 cents, so this kind of stores is called "five and dime store", or "five-and-ten store". At present, there are "liang3 yuan2 dian4" in many cities in China. "liang3 yuan2 dian4" means the price of the goods is two yuan, or the goods are very cheap. Ordinary people think the goods in this kind of stores are very valuable, so they will not buy one only. The same kind of stores named in different ways reflects the different national mentalities. Chinese people don't want to be considered stingy, five cents, one jiao maybe too tiny. So although there are coins of five cents, one jiao, the sellers will never name their stores as " 5 cents 1 jiao store". The Chinese like even number, so "two yuan" is chosen to show the goods are quite valuable. On the contrary, The English nation has pragmatic mentality, "five-and-dime store" tells people: this store offer real benefits to customers. Although the goods in "five-and-dime store" cost more than five cents and one dime, this expression is still used until today. So the different numbers used to express the same concept are related to the nation's mentality.

(5) One in a thousand

bai3 (hundred) li3 tiao1 yil (choosing one from one hundred)

From the literal meaning, "one in a thousand" means "choosing one from a thousand", which actually refers to excellent people or thing. In English the idiom can be expressed as "one in a million". In Chinese it is expressed as "bai3 li3 tiaol yil" (choosing one from one hundred). The two idioms choose different numbers, but they can express the same meaning of "extraordinary". In the two numerical idioms, no matter "bai3" (hundred) in Chinese and "thousand" in English, they are all of exaggeration, not concrete. One point should be noticed is that Chinese "bai3" (hundred) is expressed in English "thousand", in which the degree of exaggeration is higher than that in Chinese. This actually reflects the different modes of thinking. The Chinese advocate moderation whereas the English nations intend to create something new and original.

When this happens in language, larger numbers are chosen for exaggeration.

The same examples include "one and only/ first and last/ du2 yil wu2 er4 (two)"; "think twice before you leap/ san1 (three) si1 er2 hou4 xing2"; "It's the first step that is troublesome/ wan4 (ten thousands) shi4 kai1 tou2 nan2"and so on.

\section{B. Numerical Idioms with Equivalent Expressive Structures but Different Expressive Means}

On the basis of the equivalence of connotative meaning, the expressional structures are corresponding, but the expressive means are different.

\footnotetext{
${ }^{2}$ Here the two characters together with an underline are regarded as a whole in meaning.
} 


\section{Adoption of the same number}

The contents expressed by both English and Chinese numerical idioms are equivalent, the expressive structures and the chosen numbers are quite corresponding, but the difference lies in the different expressive means. For example:

(6) two strings to one's bow

liang3 (two) shou3 zhun3 bei4 (two kinds of preparations)

"Two strings to one's bow" and "a second string to one's bow" refer to the meaning that the English longbow men all have the second string to change the first one when it is broken. Even the common archer also takes another bow for preparation (Zhuang, 2002). Therefore, people often use the two idioms to compare "two kinds of preparations". (This means if the first try loses, there is still the other method). This idiom comes into being from life experience. The Chinese numerical idiom "liang3 shou3 zhun3 bei4" is originated from the slogan "yil ke1 hong2 xin1, liang3 shou3 zhun 3 bei4" before Chinese Culture Revolution. "yil ke1 hong2 xin1" means the ideal of revolution to climb onto the peak of culture and science in order to realize the socialist modernization. "liang3 shou3 zhun3 bei4" refers to: one choice is to pass the university entrance examination, the other choice is to study by oneself at home if lose. Afterwards, this idiom is considered as "two kinds of preparations", that is, two different solutions. This Chinese numerical idiom is an extension of the historical cultural background of our nation's special period.

(7) One rotten apple spoils the whole barrel

yi1 (one) ke1 lao3 shu3 shi3 huai4 le yi1 guo1 (whole) zhou1

From the literal meaning, the idiom "rotten apple" means the apple is rotten; the extended meaning refers to a bad guy, individual who brings disgrace to his group. The sentence means, one bad guy will give the whole group bad effect. In Chinese idiom "Yi1 (one) ke1 lao3 shu3 shi3 huai4 le yi1 guo1 zhou1", the mouse's stool is compared to the thing that will damage the whole group, and the bad influence to the group is stressed. Because of the different life experiences of both nations, the two idioms describe the tenors through similar association and express the same meaning by using different vehicles.

(8) a straw shows which way the wind blows

yi1 (one) ye4 zhi1 qiu1 (we know the upcoming of the autumn from a piece of leaf)

The literal meaning of the idiom "a straw shows which way the wind blows" is that from one straw we know the direction of the wind. That means we can expect the future of something or the trend of something from details. The idiom "yil ye4 zhil qiul" means one may know the autumn is coming from a falling leaf. In the two idioms, "a straw" and "ye4" (a leaf) all mean details. The number "a" and "one" are cognates sharing the same meaning, which are coincide with the Chinese number "yi1". Because of different experiences, the two idioms use "wind" and "qiul" (autumn) to refer to the near future.

\section{(9) Seven Stars}

Bei3 dou3 qi1 (seven) xing1 (the stars' name)

The numerical idiom "seven stars" is originated from Greek mythology, Athoras and Pulitzer had seven daughters. They became seven stars after they died, which formed "seven stars". This numerical idiom is associated with Greek mythology. In Chinese, the idiom "Bei3 dou3 qi1 xing1" derived from the observation of the astronomical phenomena of the seven stars in the sky. "dou3" was an ancient measuring tool. This tool is made of board in a form of a square object with a handle with the top mouth slightly larger than the bottom. The shape of the seven stars in the sky is just like that of "dou3", and then the numerical idiom is used to express "Seven Stars."

There are similar examples, such as "It' s along story/ yi1 (one) yan2 nan2 jin4" (one can not tell a story from one sentence); "birds of a feather/of a kind/ yil (one) qiul zhil he2" (refer to the same kind of persons, often have negative meaning); "three women and a goose make a market/ san1 (three) ge4 nv3 ren2 yil (one) tai2 xi4" (three women are adequate to give a piece of opera); "one boy is a boy, two boys half a boy, three boys no boy/ "yil (one) ge4 he2 shang4 tiao1 shui3 chi1, liang3 (two) ge4 he2 shang4 tai2 shui3 chi1, san1 (three) ge4 he2 shang4 mei2 shui3 chi1" (one monk carries water by himself, two monks carry water together, three monks do nothing) and so on.

\section{Adoption of the Different Number}

The contents expressed by both English and Chinese numerical idioms are equivalent, the expressive structures are quite corresponding, but the difference lies in the different expressive means and the choice of numbers. For example:

(10) in for a penny, in for a pound

yi1 bu4 zuo4, er4 (two) bu4 xiu1 (you will never stop if you start to do something)

The two idioms have the meaning of "Once we have started, we must carry on to the end". The literal meaning of "in for a penny, in for a pound" is betting against a penny is the same to betting against one dollar. Actually it means if you start to do one small thing, you should continue to do it. This idiom has the meaning "you'd better do something no matter how hard it is" or "no matter what difficulties you may face, you should see through". The focus is on "never stop half way". The Chinese idiom "yi1 bu4 zuo4, er4 bu4 xiul" means once you start to do something, you'd better do it until to the end. The focus of this idiom is on "never stop". This is a summary of the armed rebellion of Zhang guangzui which had no ending in Chinese history.

(11) Two heads are better than one.

san1 (three) ge4 chou4 pi2 jiang4 ding3 ge4 zhu1 ge3 liang4 (three cordwainers have the same talents to Zhu Geliang) 
The two idioms are all proverbs which indicate two heads are better than one. Actually it means you'd better ask for other people's advice and accept other people's help when you are in trouble. Here the number "two" is imaginary, means "many". This idiom is just a summary of simple living experience. The Chinese numerical idiom "san 1 ge4 chou4 pi2 jiang4 ding3 ge4 zhu1 ge3 liang4" is full of the color of Chinese culture. As we all know, Zhu Geliang, who was a famous person in Chinese history, is a symbol of wisdom in Chinese culture. But most English and American people may not know who he was and what the relationship between "chou4 pi2 jiang4" (three cordwainers) and him is. In this idiom cordwainer means common people. The number "three" is imaginary, means "many". This proverb means although what cordwainer does is simple work, but if many cordwainers cooperate with each other, they will have many solutions, and their ability is stronger than Zhu ge liang. So if people are in trouble, there are many solutions to the problems if they discuss together.

(12) ride Bayard of ten toes

zuo4 shi2 yi1 (eleven) lu4 che1 (take number11 bus)

The idiom "ride Bayard of ten toes" is an expression of jocosity, meaning going on foot. Here "Bayard" is the Pegasus of Romantic period, so "Bayard of ten toes" refers to a horse with two feet. Horse was the main vehicle of the early English people, then it was used for entertainment. So horse refers to every aspect of life, and sometimes refers to people. But in the Chinese culture it refers to the talented person not the common one, so misunderstanding will arise. Here "Bayard of ten toes" refers to people, so riding by such horse means walking on foot. In Chinese, the idiom "zuo4 shi2 yi1 lu4 che1" also refers to "going on foot", because two legs just like the number "11". This reflects that Han nationality is very good at making jocosity.

(13) five fingers

san1 (three) zhi1 shou3 (three hands)

"Five fingers" refers to stealer, because one film named "five fingers" tells a story about a big stealer. This film was very popular before and after the year 1950. Thus the "five fingers" came into being. In Chinese "san1 zhi1 shou3" is known by everyone, which refers to stealer. In Chinese there is an original character pronounced as "pa2" used to refers to "san1 zhi1 shou3" (three hands), which means stealer who often steal other people's belongings. This character "pa2" and the Chinese idiom "san1 zhil shou3" reflect the richness of both Han nationality and the imaginable thinking of Chinese characters.

(14) It takes two to tango.

yi1 (one) ge4 ba1 zhang3 pai1 bu4 xiang3 (one palm makes no sound)

Basically speaking, the lack of the understanding of "tango" in Chinese culture easily causes the misunderstanding of the English numerical idiom "it takes two to tango". Maybe we think from the literal meaning, tango should be danced by two persons, from which unison should be analogized. But it is not the fact. First, Tango is usually danced by a man and a woman. The bold and straightforward feature of man and the flirtatiousnes of woman constitute the tremendous tension of tango. The moral "temptation, erotic" of this idiom is easy for people to think of its homophony tangle. "it takes two to tango" actually uses the partial tone of "tangle". The whole idiom actually is created from the English idiom "It takes two to make a quarrel". The meaning is that if two persons quarrel with each other, the mistake is not one's but that of the two. This meaning comes from people's entertainment in English culture. The literal meaning of Chinese idiom "yi1 ge4 ba1 zhang3 pail bu4 xiang3" is that one cannot make sound if just flap one palm in the air. Flap both palms will make sound. Here the palm refers to people. This means conflict is not just made from one side. Both sides take responsibility. This idiom comes from the people's experience of life.

The similar examples are "the whole nine yards/ yi1 (one) ju3 cheng2 gong1" (succeed at last); "An eye finds more truth than two ears/ bai3 wen2 bu4 ru2 yi1 jian4" (seeing is better than hearing); "Rome was not built in one day./ bing1dong4 san1 chi3 fei1 yil ri4 zhi1 han2" (the ice frozen for three feet is not because the cold weather lasts for one day); "Once bitten, twice shy/ yi1 zhao1 bei4 she2 yao3, shi2 nian2 pa4 jing3 sheng2" (people will be afraid of rope if once bit by a snake)and so on.

\section{Numerical Idioms with Different Expressive Structures but Equivalent Expressive Means}

On the basis of the equivalence of connotative meaning, the expressive structures are different, but the expressional means are corresponding.

1. Adoption of the Same Number

The contents expressed by both English and Chinese numerical idioms are equivalent, the choice of numbers and the expressive means are quite corresponding, but the difference lies in the different expressive structures. For example:

(15) hundred and hundred

chang2 ming4 bai3 (hundred) sui4 (live for one hundred years)

The idiom "hundred and hundred" is a fine blessing, means continuing living for another one hundred years. So this blessing is imaginary, not definite without limitation of life span. But in ancient china, it was so difficult to live long. So the idiom "chang2 ming4 bai3 sui4" (live for one hundred years) means "living for a long time". The two numerical idioms in both England and China all reflect the ideal of living for a long time from ancient times.

(16) second to none

shou3 qu1 yi4 (one) zhi3 (the thumb bends)

The meaning of the idiom "second to none" refers to the best one, not worse than anybody else. In the Chinese idiom 
"shou3 qu1 yi4 zhi3", "shou3" means the first. This idiom means if one counts something by using fingers, he has to bend the thumb, which refers to the first one. The extending meaning also refers to the best one. The two numerical idioms reflect the differences of the thinking mode of people between Chinese and Western countries. The Chinese culture forms a thinking mode that stresses the intuitive intention, which experiences, feels and grasps the object through intuition. The western people emphasize a thinking mode that stresses analysis of and is biased towards abstract things.

(17) A penny saved is a penny gained.

sheng3 yi1 (one) fen 1 shi4 yi1 (one) fen 1 (a cent saved is a cent earned)

The literal meaning of the idiom "A penny saved is a penny gained" is that saving a penny means gaining a penny, that is, saving money is very clever. Do not look down up small amount of money. The Chinese numerical idiom "sheng3 yi1 fen 1 shi4 yil fen1" means all money come from saving. Because our monetary unit is different, in English the smallest monetary unit "penny" is used whereas in Chinese "cent" is used. Although the two numerical idioms does not equate in structure, from the two idioms we can see both English and Chinese people like saving.

The similar examples are " be of one heart and one mind /of one mind/ yi1 (one) xin1 yi1 (one) yil (one heart one mind); "five by five/ wu3 (five) duan3 shen1 cai2" (squat); one's every move/ yi1 (one) ju3 yil (one) dong4 (the movement and activity); "make the best of both world /have things both ways/ liang3 (two) quan2 qi2 mei3" (consider every aspect perfectly)and so on..

2. Adoption of the Different Number

The contents expressed by both English and Chinese numerical idioms are equivalent, the expressive means are quite corresponding, but the difference lies in the different expressive structures and the choice of numbers. For example:

(18) one foot in the grave

ban4 (half) jie2 ru4 tu3 (half the body has been buried in the earth)

The idiom "one foot in the grave" is mostly translated as "one foot has already been in the grave", of which the metaphoric meaning is very clear. It has humorous style, means "near to death" and "cannot live for a long time". This idiom initially related to the figure Charon in ancient Greek myths. He is the god who could carry the revenants from the Styx to the hell (Zhuang, 2002). The idiom "with one foot in Charon's ferryboat" lately changed into the present numerical idiom "one foot in the grave". In Chinese the idiom "ban4 jie2 ru4 tu3" means half of the body has already been in the earth indicating the person will die soon. The two idioms respectively use "one foot "and "ban4 jie 2 shen 1 zi" to refer to the people's physical condition. The two idioms all use vivid rhetorical device of metonymy. This reflects that both Chinese and English nations have the habit to refer to the whole by using the part.

(19) crack up (flatter / honor / praise) to the nines

shi2 (ten) quan2 shi2 mei3 (very perfect)

In English cultural tradition, the number "nine" is one of "God Numbers" in people's minds. For Westerners, "nine" has symbolic meaning of "Divinity" and "sacred thing to peak". The first meaning of "Nine" is its self-multiplier of "three". "Nine" is the symbol of truth in Hebraic culture, and represents the absolute order of all things in Christianity. "Nine" is a mysterious spirit figure in religion. We often find the number "nine" in ancient Greek mythology. In addition, according to British superstition cats have nine lives, so the number "nine" means strong vitality. And many rental contracts are set 99 years. Compared with Chinese, "nine" does not have the meaning the supreme and honor , but it also presents "many", "perfect", "happy", "long" and others. At the same times, according to numerology, "three" is "Trinity" and on behalf of a perfect whole, "six" is two times of "three" and "shuang1 chong2 wan2 mei3" (the perfect dual), and "nine" is three times of "three" and "Duo1 chong2 wan2 mei3" (many aspects are perfect). The phrases of "Crack up (flatter / honor / praise) to the nines" is born and also means perfect. The numerical idiom reflects a specific meaning of "nine" in myth and religion. The Chinese culture regards "shi2" as a symbol of perfection originating from its grapheme. Shuo Wen Jie Zi (a Dictionary of Chinese Hieroglyphics and Words) explains the two strokes of "shi2" respectively, "the horizontal stroke representing east and west, the vertical stroke representing south and north, four directions and their centers are all present." So "shil" symbolizes totality, completeness, perfection and summit. Meanwhile, "shi2" is a figure of earth with Yin attribute. Dong Zhongshu summarized Yin and Yang (the two opposing principles in nature), three talents (heaven, earth and people) and five elements (wood, fire, earth, metal and water) as "shi2 da 4 tian1 shu4"(ten predestinate figures) according to the theory of harmony between man and nature. Meanwhile "shi2" is a figure of heavenly stems, playing an important role of conversion and progression in Chinese calendrical system. Nevertheless, the figure "shi2" bears the closest relations to ten-figure arithmetic, and the concept of decimal system has filtered deep into people's minds. "shi2" has become the figure of extremity, indicating totality, completeness and ultimacy.

(20) Every Tom, Dick and Harry

zhang1 san1 (three) li3 si4 (four) (two common names of Chinese people)

Here "Tom, Dick and Harry" are the most common names in English, generally refer to common people, like "zhang1 san1, li3 si4, wang2 er4" (most common Chinese people's names). But in usage, this expression has the sense of defiance, especially refers to "very very plain, without doing any important thing." Because "zhang1" and "li3" are the most commonly used family name, in order to give example easily, the familiar family names and numbers are selected together to refer to the entire part. So "zhang1 san1 li3 si4" are supposed names, generally refer to some 
common people. The two idioms all have strong color of nation.

There are some other similar examples, such as "be of two minds/ san1 (three) xin 1 er4 yi4", "in one or two words/ san1 (three) yan2 liang3 yu3", "twenty and twenty times/ san1 (three) fan1 wu3 (five) ci4", "leave no stone unturned/ qian1 (thousand) fang1 bai3 (hundred) ji4", "One barking dog sets the whole street barking./ yi1 quan3 fei4 ying3, bai3 (hundred) quan3 fei4 sheng1)" and so on.

\section{Numerical Idioms with Different Expressive Structures and Means}

On the basis of the equivalence of connotative meaning, numerical idioms have the differences not only in the expressive structure, but also in the expressive means.

1. Adoption of the Same Number

The contents expressed by both English and Chinese numerical idioms are equivalent, the expressive means and structures are not corresponding, the sameness lies in the adoption of the same number. For example:

(21) as poor as a church mouse

yi1 (one) pin2 ru2 xi3 (as poor as being washed)

The idiom "as poor as a church mouse" has something to do with the religion believed by Westerners, in which the churches are the places where the followers take religious activities and devoutly worship. Surely nobody eats there and there is no food cupboard. Thus the churches are quiet with solemn. Therefore, it is absolutely impossible to find something to eat for mice in churches. A church mouse is very poor, so the idiom "as poor as a church mouse" comes into being, which is used to describe extremely poverty (Zhuang, 2002). "yi1 pin2 ru2 xi3" refers to the situation that someone is poor like being washed with water without owing a penny, which is the performance of the visual image thinking emphasized by the Chinese nation.

(22) A 1, A-one

tian1 zi4 di4 yi1 (No.1) hao4 (first-rate)

Loyd's Register of Shipping, which is established in England in 1760, has published Lloyd's Shipping Yearbook, which proposes the tonnage and grade of the merchant ships registered. The levels of hull quality are indicated with alphabetical order, and the merits of equipment are shown with numbers. The ships classified as the first level and grade are set A1. Dickens, who was a famous British writer, firstly applied A1 to any thing and people in 1837 to symbolize "first-class" or "excellent". A1 is considered as A-1, A-one (Zhuang, 2002). In the past, the Chinese used to show the order with Thousand Characters in which "Tian" (heaven) is the first word of the sentence "World yuen wong (tian1 di4 xuan2 huang2)". It refer to first or the first number in grade one, and represents the highest, biggest or strongest. So "A-one" means "No. one", that is, A1 in present sort (Ma, 2004).

(23) hang by a hair / thread

qian1 jun1 yi2 (one) fa4 (thousand "Jun" were hung with one hair)

The idiom of "hang by a hair / thread" refers to the scabbard sword tied with a thin thread (or mane) above the head and will fall down anytime. According to literal meaning, the translation of the idiom is hanging with a thin thread (or horse's mane) to show "at risk" or "shaky". The numerical idiom has correspondence with Greek legend, in which Dionysius, who was a tyrant king of city-state of Syracuse in Sicily Island, gave a warning to Damocles that the kings were on the thrones, but they also feared every day and the loss of life may be imminent at any time. So the meaning has no difference with the idiom "qian1 jun1 yi2 fa4" in Chinese. "Jun" was an ancient unit of weight, one "Jun" have thirty "Jin" in Chinese. The idiom that thousand "Jun" were hung with one hair, was shown that the situation was extremely emergent or very critical, which is used to present that the people or something are at risk. This kind of idioms is the summary of the real experience in daily life.

There are some, like "fall between two stools / liang2 (two) tou2 luo4 kong1", "not know one end of sth. from the other / yi1 (one) qiao4 bu4 tong1", "play second fiddle / er2 (second) ba3 shou3", "with one mouth / yi1 (one) kou3 tong2 ci2", etc.

2. Adoption of the Different Number

The contents expressed by both English and Chinese numerical idioms are equivalent, but the expressive means, structures and the adoption of the number are different. For example:

(24) nine days' wonder

tan 2 hua1 yi1 (one) xian4 (good things or sights disappear soon after their brilliant appearance)

"Nine days' wonder" is generally defined as "the things which incur much attention in a short time is forgotten immediately, or "the night-blooming cereus". But according to literal translation, it represents "nine-hit wonders". There is a history with nine days to express a moment. "Nine days' wonder" is said to be derived from an ancient Roman custom. When there were abnormal phenomena in nature, Romans would regard it as an ominous sign. In order to do with disasters, Catholic Church always held a religious prayer ceremony that lasted for nine days. But no body would talk about it when the ceremony was over. So based on the Western customs, "nine days' wonder" means rare things, or a prominent man during a specific time who disappears after his influential presence (Zhuang, 2002). In Chinese, the number idiom "tan2 hual yil xian4" is born from the special case of night-blooming cereus. When the flowers are blooming, the spendthrift slowly tilts, and the purple cloak gradually opens, then the big flowers with more than 20 petals which are white just like snow bloom. When blooming, the petals and stamens are quivering with dazzling beauty. But the flowers last for only 3-4 hours, then the corolla will close and finally quickly wither away. So the idiom implies 
that the good things or sights disappear soon after their brilliant appearance.

(25) in seven-leagued boots

yi1 ri4 qian1 (thousand) li3 (very fast)

The idiom "in seven-league(d) boots" is related to the ancient folk tales of United Kingdom. It is said that a pair of God boots could walk Seven $\mathrm{Li}$, which is about 21 miles a foot. Therefore, the idiom is applied to represent a very fast speed. The Chinese idiom is related to common life experience, "yil ri4 qian1 li3" means the horse run fast and can run a thousand Li in a day, which now describes a person who makes a big progress very quickly or a thing that has a rapid development. In this case, the rhetorical device of exaggeration is employed.

(26) in two shakes of a lamb's tail

yil (one) tan 2 zhi3 jian1 (time goes by immediately)

Lambs and other animals always shake their tails before running. It is said that the time spent on two shakes of their tails is less than that of many other animals' one shake of tail. The idiom "in two shakes of a lamb's tail" comes from the characteristic of the lamb. With literal translation, it refers to the moment of the lamb's tail shaking twice. Generally, it is used for figurative meaning which only means "soon or immediate". It is believed that the idiom "in two shakes of a lamb's tail" might be expanded from its synonymous phrase "in two shakes" which preciously appeared in the English language and might derive from the gambling with dice-throwing. Originally it is written as "in two shakes of a dice box" (Zhuang, 2002). Because Britain is a country abundant in sheep which is its pillar industry, the British have more observation of sheep and application to idioms. "yil tan2 zhi3 jian1" is originally used in Buddhism language in China, which means "sixty years have passed when one figure shakes". The time of finger shaking implies the very short moment. Because China is a big Buddhist country and the Buddhist teachings enjoy popular support, many numerical idioms cited are associated with this aspect.

(27) At sixes and sevens

luan4 qi1 ba1 (eight) zao1 (be in a mess)

The idiom "At sixes and sevens" is a common verbal phrase and has a long history. It is generally believed that it is relevant to dice-throwing which is a gambling jargon. The shape of dice is a cube and the numbers of points on each surface of which are from one to six. However, it is said that the highest number of point should be more in Middle Ages. There are six points and seven points. So thirteen points are ideal, which is possible with six and seven point together. But it is difficult. The people, who were upset or in confusion, would try to make a bet. The idiom "at sixes and sevens" is firstly used to express the meaning "only to stake at the highest point (that is, six or seven points)" or "random bet", then represents "upset mind", and finally deduces the implication of "mess", "disagree", etc (Zhuang, 2002). Chinese often use the idiom "luan4 qi1 ba1 zao1" to describe the things disorganized and difficult to do with. The idiom is related to historical events. There was a "Rebellion of Seven States" under the domination of Emperor Wen in Western Han Dynasty, Which is the origin of the expression "Qi luan". Under the domination of Emperor Si Mayan in the early years of Western Jin Dynasty, there was a "Riot of Eight Royal Highness", which lasted much longer than "Rebellion of Seven States" and seriously weakened the dominant power of Western Jin Dynasty. People suffered more severe disasters as well. So we call it "Ba zao" (Ma, 2004). Hereafter, the idiom "luan4 qi1 ba1 zaol" is produced by associating the two events, which means "the situation without order" or "be in a mess".

(28) Behind the eight ball

si4 (four) mian4 chu3 ge1 (critical environment or isolated distress)

The idiom "Behind the eightball" is related to the game "billiards" which has various forms and names. There is game "rotation" named Kelly pool, which requires player to shot 15 balls into the bags in numerical order. The player will lose points if the any ball hit the black eightball. So when the cue ball or the one which should be hit lies just behind the eightball, the player will be at a disadvantage and it is difficult to win. Accordingly, the idiom "behind the eightball" means "in a bad situation", "distress" or "frustrated" (Zhuang, 2002). "si4 mian4 chu3 gel" is a piece of Chinese allusion, which describes the situation of being attacked on all sides and isolated without any help. The story was about Xiang Yu, who heard the Chu's songs all around and felt surprised and frustrated, committed suicide because of failure at last. Since then, the people described the situation suffered whole attacks, critical environment or isolated distress with this idiom.

There are similar examples, such as "hit / knock for six / dang1 tou2 yi1 (one) bang4", "chuck up the bunch of five / liang3 (two) tui3 yi1 shen1", "with a grain of salt / ban4 (half) xin4 ban4 yi2", "a close call / jiu3 (nine) si3 yi1 sheng1" and so on.

\section{CONCLUSION}

The comparison and contrast between English and Chinese idioms reveals two points: universality and individuality. The universal implications in English and Chinese idioms stem from our agreements on the universality that exists in mankind himself and the external conditions on which he relies, and even in the social and cultural backgrounds of human beings. The universality of English and Chinese idioms is reflected in the shared attributes of English and Chinese rhetoric. But different nations have different living circumstances, material cultures, social cultures, religious cultures and historical development as well as varied thinking modes in perceptions and preferences so that they observe and experience all the happenings of the realistic world from different perspectives in different ways and form 
their distinctive viewpoints and perceptions. This kind of national individuality determines the distinct characteristics of numerical idioms. The individualities of English and Chinese numerical idioms are given display in the asymmetrical phenomena in English and Chinese imagery of rhetoric.

The national cultural characteristics displayed in English and Chinese numerical idioms with equivalent connotations stem from their respective cultures including their distinct histories, thinking modes, religious beliefs, customs, social constitution, values, living styles, etc. The differences are reflected not only in the numerical idioms, but also in their rhetoric. Different religious beliefs have bestowed strong national individuality on the implications of some numbers. In terms of culture, the west has long been influenced by religions while our country has been under feudal rule for quite a long time. Different implications of numerical idioms also arose from different perceptions that the ancestors of different regions obtained on same things in their practical cognitive process throughout human history. Naturally these idioms contain different images, reflect different cultural values and become the fruits of wisdom accumulated in a nation's life experience, history and culture. In terms of different living styles, people's perceptions are indispensable from their own observation and daily life experiences, which can find display in the fact that all perceptions begin with mankind self-recognition. Human beings firstly took the experiences of their own body as the basis, speculated a scene and composed a picture, an image schema providing a basis for abstract thinking and constituting a chief medium for comprehending abstract concepts and conducting abstract thinking. Therefore, the English and Chinese numerical idioms with equivalent connotations have adopted diverse expressive forms and rhetorical devices.

\section{REFERENCES}

[1] Adam, M. (1972). Idiom structure in English. The Hague: Mouton.

[2] An, M. H. (2008). A Comparison of English and Chinese Numerical Idioms. Jilin: Yanbian University Press.

[3] Ma, J. J. (2004). Source of Idioms. Beijing: China Civil Aviation Press.

[4] Miller, A. I. (1996). Insights of genius. New York: Springer-Verlag.

[5] Newmark, F. (1981). Approaches to translation. Oxford: Pergaman Press.

[6] Nida, E. A. (1999). Language, culture and translating. Shanghai: Shanghai Foreign Language Education Press.

[7] Parkinson, D. (2007). Oxford Idioms Dictionary. USA: Oxford University Press.

[8] Peter, A. (1981). Approaches to translation. Oxford: Pergaman Press.

[9] Pullum, G. (1987). On the definition of word. Cambridge, Mass.: MIT Press.

[10] Raymond, W. G. (1993). Why idioms are not dead metaphors. Hillsdale, NJ: Lawrence Erlbaum Associates.

[11] Zhao, A. G. \& Y. M. Jiang. (2003). Introduction to Applied Cultural Linguistics. Shanghai: Shanghai Foreign Language Education Press.

[12] Zhuang, H. C. (2002). The origin of English Idioms. Shanghai: Shanghai Foreign Language Education Press.

Yuan Kong received her M.A. degree in linguistics. She is currently a lecturer at the Department of Foreign Languages, Jining Medical University, Jining, Shandong, China. She teaches various subjects including: English intensive reading, English extensive reading, listening, nursing reading and so on. Her research interests mainly include English language teaching, applied linguistics, cross-cultural communication, etc. 